\title{
Within-set discriminations in a consistent mapping search task
}

\author{
JOHN M. FLACH \\ The Ohio State University, Columbus, Ohio
}

\begin{abstract}
This study investigated subjects' ability to make discriminations within the target and distractor sets following consistent mapping training in a visual search task. Differences in the patterns of reaction time data obtained with and without the requirements to make discriminations within target and distractor sets suggest that automatic processing entails two processing changes. One change involves treating the target set as a single equivalence class. This increases efficiency of the memory comparison process. A second change involves inhibition or filtering of distractor elements, which increases the efficiency of visual search.
\end{abstract}

The qualitative performance shifts that accompany the development of skill have been discussed by a number of researchers (Fitts \& Posner, 1967; Kimble \& Perlmuter, 1972; Laberge, 1973; Posner \& Snyder, 1975; Schneider \& Shiffrin, 1977). Early performance in a task is described in such terms as conscious, voluntary, controlled, limited capacity, and serial. Later performance, following skill development, is described as unconscious, involuntary, automatic, unlimited capacity, or parallel. This paper examines this qualitative performance shift in the context of a visual search task.

Schneider and Shiffrin (1977; Shiffrin \& Schneider, 1977) proposed that two control processes govern performance in a visual search task-controlled search.and automatic detection. Controlled search is described as a slow, serial process in which reaction time is a monotonically increasing function of the number of comparisons that must be made (load). Automatic detection, on the other hand, is described as a rapid, parallel process in which reaction time is independent of the number of comparisons. Two experimental factors determine whether performance is governed by controlled search or automatic detection-the amount of practice and the type of stimulus mapping (consistent vs. varied).

With a varied stimulus mapping, targets and distractors are chosen from a single set of stimuli such that an item that is a target on one trial may appear as a distractor on another trial. Whenever a varied stimulus mapping

\footnotetext{
This research was submitted to the Graduate School of The Ohio State University in partial fulfillment of the requirements for the $\mathrm{PhD}$ degree. Financial support for this project was provided by The Ohio State University Graduate School through the Graduate Research Award and by the Psychology Department through the Toops Award. I gratefully acknowledge the assistance of Sandy Loeb in software development and Merry Northrup in data collection. I also thank Richard Jagacinski, Harvey Shulman, Lester Krueger, and Mari Jones for comments on earlier drafts of this paper. Requests for reprints should be sent to John Flach, who is now in the Engineering Psychology Program, University of Illinois, 144 Mechanical Engineering Building, 1206 W. Green St., Urbana, IL 61801 .
}

is employed, performance will be governed by controlled search independent of the amount of practice.

With a consistent stimulus mapping, targets and distractors are chosen from two disjoint sets of stimuli such that a target item will never appear as a distractor. When a consistent mapping is employed, controlled search tends to dominate early in practice, but automatic detection develops and dominates late in practice.

Following practice in a consistent-mapping visual search task, subjects become extremely efficient in discriminating among items drawn from the disjoint target and distractor sets. Subjects can decide rapidly whether a target is contained in a search display, and decision time is independent of both the number of distractors in the display and the number of targets in memory (Schneider \& Shiffrin, 1977; Shiffrin \& Schneider, 1977). The experiments reported in this paper focus on the nature of automatic detection. The specific question addressed by the experiments was: How does this ability to make rapid discriminations between targets and distractors interact with the ability to make discriminations within the target and distractor sets?

\section{EXPERIMENT 1}

Experiment 1 focused on the ability of subjects to make discriminations within the target set following the development of automatic detection. When a subject responds that a target is present in the visual display, is the decision based on gross categorical or feature distinctions between targets and distractors or has the specific target been fully identified?

To answer this question, subjects were first trained in a standard 2-response, consistent mapping, visual search task. In this task, subjects were instructed to indicate whether a target was present or absent and to respond as quickly as possible. Following extensive consistent practice, the subjects were transferred to a new task. This transfer task was identical to the original task in all respects except for the response demands on positive 
trials. For the transfer task, the target set was divided into two equal subsets. On positive trials (target present), the subjects were required to choose between two alternative responses, each corresponding to one of these two target subsets. Thus, subjects were trained with a 2-response task (no target, target) and were transferred to a 3-response task (no target, target [subset 1], target [subset 2]). In the training task, subjects were required to discriminate only between targets and distractors (nontargets). In the transfer task, subjects were faced with the additional requirement to discriminate between target subsets.

The performance signature for identifying the development of automatic detection in the typical two-choice (no target, target) visual search task is independence of reaction time from memory set size (the number of candidate targets on a given trial) and display size (the number of items on a visual display). The aim of Experiment 1 was to determine whether this independence from display and memory set size would be affected when subjects were transferred to the 3-response task with its additional within-target-set discrimination requirement.

What pattern of interactions might result from this manipulation? At one extreme, it has been argued that independence of reaction time (RT) from memory-set size is totally dependent either on the ability to "categorize" targets and distractors (Jonides \& Gleitman, 1972, 1976) or on the ability to focus on invariant physical differences between targets and distractors (Gibson, 1969). This position will be referred to as the partial-analysis hypothesis, since the pattern of performance associated with automatic detection is attributed to the ability to focus on a few key diagnostic attributes of stimuli for discriminating targets from distractors and the ability to ignore all other attributes. Such a strategy would result in a blurring of distinctions within the target and distractor sets that should interfere with the ability to make within-set discriminations (Gleitman \& Jonides, 1976). Results from Experiment 1 that showed independence of RT from memory set and display size at the end of practice with the 2-response task but dependence of reaction time on memory set and display size following transfer to the 3response task would support this hypothesis.

At the other extreme, it has been argued that automatic processing (independence of RT from memory set and display size) is relatively independent of the ability to focus on categorical and feature distinctions (Shiffrin \& Schneider, 1977). In Shiffrin and Schneider's original model, it was assumed that encoding of display items was parallel, with all items receiving full perceptual analysis. This will be referred to as the full-analysis hypothesis. With this model there is no a priori reason to expect interference between automatic processing and the requirement to discriminate within the target set. Results from Experiment 1 that showed independence of RT from memory-set size and display size, both at the end of practice with the 2-response task and following transfer to the 3-response task, would support this full-analysis hypothesis.

These two extreme positions, the partial- and the fullanalysis hypotheses, do not differentiate between the memory-set-size and the display-size effects on performance. Reaction time is considered to be a function of "load" (Schneider \& Shiffrin, 1977), that is, the number of comparisons (memory set size $\times$ display size) required. A third possible outcome for Experiment 1 was that the additional discrimination required by the 3response task would interact only with display size or only with memory-set size. Such a result would indicate that despite the similarities between the interaction of practice with memory-set size and the interaction of practice with display size (slope of reaction time function decreasing with practice in both cases), the underlying cognitive processes might be different. Thus, automatic detection might not be a single control process as described by Shiffrin and Schneider (1977), but might actually be two processes-one mediating the effects due to memory-set size and the other mediating the effects due to display size. For example, categorical or feature distinctions might permit a parallel search of the display to locate a target, but a serial memory comparison process might be required to determine to which subset the target belonged. This hypothesis will be referred to as the dual-analysis hypothesis.

\section{Method}

Subjects. Six male and 2 female undergraduates from the introductory psychology subject pool at The Ohio State University served as subjects. The subjects were given class credit for up to $4 \mathrm{~h}$ of participation and were then paid at the rate of $\$ 2.50 / \mathrm{h}$ for all additional hours. All subjects had normal or corrected-to-normal vision. All subjects were right-handed.

Design. This experiment employed a $2 \times 2 \times 2 \times 2 \times 8$ repeated measures design. The factors were task (2-response/training vs. 3 response/transfer), trial type (positive/target present vs. negative/no target), display size ( 2 vs. 4 ), memory-set size ( 2 vs. 4 ), and subjects. The dependent variables were RT and percent errors.

Apparatus and Stimuli. A Data General Nova 4 computer was used to present the stimuli and to collect the responses. The stimuli were presented using Tektronix Monitor 604 CRT displays controlled by a Megatek interface.

The subjects were seated in a small experimental booth, approximately $71 \mathrm{~cm}$ from the CRT display. Two subjects were run simultaneously in adjacent booths. Background illumination was dim but comfortable.

The visual search frame contained four stimulus positions. These positions were at the corners of an imaginary square with a fixation marker $(x)$ at the center. The visual frame subtended $1.37^{\circ}$ vertically and $1.29^{\circ}$ horizontally $\left(1.88^{\circ}\right.$ diagonally $)$. Individual stimuli were $0.32^{\circ}$ of visual angle high and $0.24^{\circ}$ of visual angle wide. The memory display position was located directly above the visual search frame. Also, an error message was displayed below and to the left of the visual frame for subjects in booth 1 and below and to the right of the visual frame for subjects in booth 2 . Neither person could see the incorrect message for the other subject, since masks covered that portion of the screen.

The visual stimuli consisted of eight letters (C, D, F, G, H, K, $\mathrm{L}, \mathrm{M})$ and eight numbers $(2,3,4,5,6,7,8,9)$. For half of the 
subjects, targets were chosen from the set of numbers and distractors were chosen from the set of letters. For the other subjects, this assignment was reversed. Letters and numbers were constructed from dot matrices.

For the 2-response task, two response keys were employed. The right key, activated by the index finger of the right hand, was used to indicate that a target was present. The left key, activated by the left index finger, was used to indicate that no target had been detected. For the 3-response task, three response keys were employed. The function of the left key (activated by the left index finger) was the same as in the 2-response task. It indicated that no target was detected. Two keys were activated by fingers of the right hand. A key under the right index finger indicated that a target from one target subset was present, and a key under the right middle finger indicated that a target from the other subset was present.

Procedure. Each of the 8 subjects participated in nine 1-h sessions. Sessions were run on consecutive weekdays, with the first session beginning on a Tuesday. For the first six sessions, the subjects performed the 2-response/training task. For the last three sessions, the subjects performed the 3-response/transfer task. The training and transfer tasks differed only in the type of response required of the subjects.

A session consisted of eight blocks of 40 trials each. There were two blocks for each of the four combinations of memory-set size ( 2 or 4 ) and display size (2 or 4). Memory-set size and display size remained constant within a block. The order of blocks for each session was chosen randomly, with the constraint that no particular combination of memory-set size and display size be repeated until all combinations had been presented once. Each block began with a READY message displayed on the screen for $3 \mathrm{sec}$. Each block was followed by a BREAK message, which was displayed for $10 \mathrm{sec}$. There was a 60 -sec break between blocks.

A single trial consisted of the following sequence. First, the memory set was displayed for $3,000 \mathrm{msec}$. Following the memory set, four pattern masks were presented for $1,000 \mathrm{msec}$. The pattern masks were positioned at the corners of an imaginary square centered around an $x$ which marked the center of the screen. There was no overlap between the spatial position of the memory set and the position of the pattern masks. The pattern masks were then replaced, for $500 \mathrm{msec}$, by stimuli drawn from the target and distractor sets. If the display size was 4 , all four masks were replaced by characters. If the display size was 2 , then characters appeared on one diagonal of the square and masks remained on the other. The diagonal used on a particular trial was chosen randomly, with the constraint that each diagonal contain stimuli equally often within a block. The targets and distractors were then replaced by masks for $1,000 \mathrm{msec}$.

To construct the memory sets for each trial, the target set was divided into two subsets-early $(2,3,4,5$, or C, D, F, G) and late $(6,7,8,9$ or $H, K, L, M)$. If memory-set size was 2 , the character in the first position of the memory display was drawn from the early subset and the character in the second position was drawn from the late subset. If memory-set size was 4 , the characters in the first (left) two memory display positions were filled using characters from the early subset and the right two memory positions were filled using characters from the late subset. Each of the eight characters in the target set appeared in the memory display an equal number of times within a block.

A single target character from the memory display appeared in the subsequent visual frame on half of the 40 trials in each block. No target was present on the remaining 20 trials. A target was chosen equally often from each of the two or four positions of the memory display. Thus, on half of the 20 positive trials, targets were drawn from the early subset and on the other 10 trials targets were drawn from the late subset. The position of the target in the visual frame was determined randomly with the constraint that a target appear equally often in each of the four display positions within a block. The remaining one or three positions were filled by characters randomly drawn from the distractor set, such that no single item was repeated in the same display. On negative trials, all two or four positions were filled by items drawn randomly from the distractor set, with two constraints. First, no item was repeated in a single display. Second, either an F or a 5 appeared as a distractor on half of the 20 negative trials. This last constraint becomes important for later experiments. The order of positive (target present) and negative (no target) trials was determined randomly for each block.

For both the 2-response and the 3-response tasks, the subjects were instructed to respond as quickly and accurately as possible. In the 2-response task, the detection of a target (positive trial) was indicated by a press on the right key and a negative trial was indicated by a press on the left key. The 3-response task was different from the 2-response task in that a different positive response was required, depending on whether the target was a member of the early or late target subset. If a trial was positive and the target was drawn from the early subset, the correct response was made by depressing a key under the right index finger. If a trial was positive and the target was drawn from the late subset, the correct response was made by depressing the key under the right middle finger. The correct response for negative (no target) trials was identical for both the 2- and 3-response tasks.

Two types of feedback were provided to the subjects. An indication as to whether a particular response was correct or incorrect followed each trial. If a response was incorrect, the word INCORRECT appeared in the lower portion of the screen. If a response was correct, the screen remained blank for $3,000 \mathrm{msec}$. In addition, at the start of each session, the subjects were shown a graph that indicated the performance of each subject in the previous sessions. Points on this graph were computed using the formula: Score $=\mathrm{RT}+50(\mathrm{ERR})$, where $\mathrm{RT}=$ mean reaction time per block, and $E R R=$ mean number of errors per block. A $\$ 5$ bonus was awarded to the subject whose performance was best (lowest score, as computed using the above formula) on the final session.

\section{Results and Discussion}

The principal comparison used the repeated measures analysis of variance described in the Design section above.

RT data was obtained in the following manner. Median RTs were computed separately for the 20 positive and 20 negative trials in each block. A block of trials represented a particular memory-set-size/display-size combination. The median RTs obtained on the last two sessions in a task (Sessions 5 and 6 for training and Sessions 8 and 9 for transfer) for a particular subject were averaged to yield a single score for positive and negative trials for each memory-set-size/display-size combination. These scores were then used in the analysis of variance. Percent error scores were combined in a similar fashion-that is, averaged across the four blocks of a particular memory-setsize/display-size combination obtained in the final two sessions of a particular task.

Reaction time. The RT results are shown in Figure 1. Each data point represents an average across 8 subjects. Analysis of variance yielded a significant three-way interaction between task, trial type, and memory set size $[F(1,7)=171.76, p<.001]$ and a significant two-way interaction between trial type and display size $[F(1,7)=$ $11.37, p<.025]$. These interactions can best be understood in the context of Tables 1 and 2 .

Table 1 shows the difference in mean RT between memory-set sizes 4 and 2 at each combination of the other independent variables - task, trial type, and display size. In the 2-response (training) task, the differences are small. Somewhat surprisingly, RT tended to be lower for 


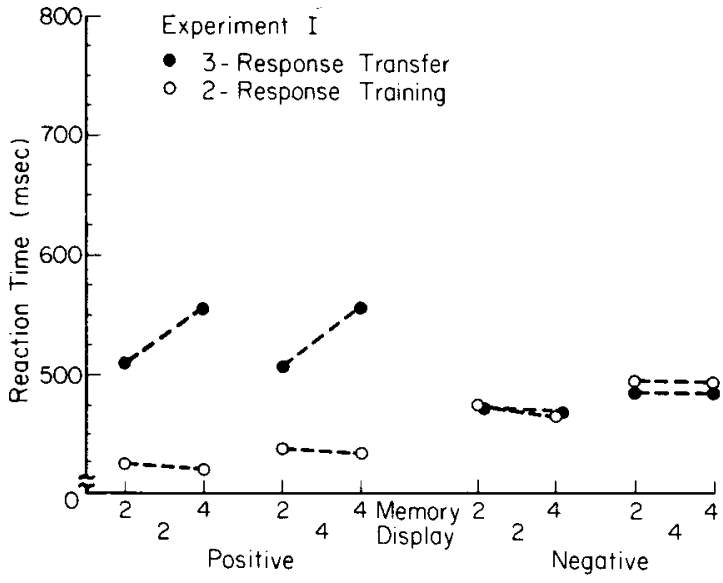

Figure 1. Reaction time data for Experiment 1. Reaction times are plotted as a function of task (3-response vs. 2-response), trial type (positive vs. negative), display size (2 vs. 4), and memory-set size (2 vs. 4).

memory-set size 4 than for memory-set size 2 . This is indicated by the negative signs. RT differences, as a function of memory-set size, are also small for negative trials in the 3-response task. However, rather large RT differences are found for positive trials in the 3-response task. These differences were found to be statistically significant using Dunn's multiple comparison procedure $[C R(.01,20,7)=21.37]$.

Table 2 shows the difference in mean RT between display sizes 4 and 2 at each combination of the other independent variables-task, trial type, and memory-set size. At the end of training in the 2-response task there is a consistent difference, such that RTs are faster for display size 2 than for display size 4 . The differences are somewhat larger on negative trials than on positive trials. Similar differences are seen for negative trials in the 3response task. However, there are only small RT differences for positive trials in the 3-response task.

The pattern of results shown in Figure 1 supports the dual-analysis hypothesis. The extra discrimination required by the 3-response task did not interfere with the subjects' ability to locate targets in the display. There was no interaction between task and display size, and the pattern of RTs obtained on negative trials was identical for the two tasks. The extra discrimination did, however, affect the memory comparison process. At the end of practice in the 2-response task, RT was independent of memory-set size. However, RT for positive trials in the 3-response task increased as a function of increasing memory-set size.

The fact that the requirement to identify a specific target (in order to determine subset membership) did not interact with display size is consistent with results found by Jonides and Gleitman (1976). They were concerned with subjects' ability to identify the particular target when a categorical difference existed between targets and distractors in a visual search task. Subjects were tested in a "modified between-category" search condition. In this condition, catch trials were introduced in which nontar- gets from the target category (numbers) were inserted in the display. Thus, to avoid getting "caught" on these trials, it was necessary for subjects to know more than that a number appeared on the display (distractors were letters). The presence of catch trials did not interact with display size. However, it did affect the intercept, suggesting that additional time was needed to identify the targets once they were located in the display. Memory-set size was not varied in this experiment.

An additional analysis of variance compared changes in RT across sessions in the 2-response/training task. There was a significant main effect for sessions $[F(5,35)=5.86, p<.001]$. Average $\mathrm{RT}$ improved from $590 \mathrm{msec}$ in Session 1 to $450 \mathrm{msec}$ in Session 6. Sessions did not interact with any of the other factors.

The reduction in RTs across sessions in the 2-response training sessions is consistent with the development of automatic detection. The fact that there was no interaction between sessions and either display or memory-set size suggests that some automatic detection was operating early in practice. This can probably be attributed to the fact that categorically distinct targets and distractors were employed in this study.

Percent errors. An analysis of variance for percent errors resulted in a significant interaction between task and

Table 1

The Difference in Mean Reaction Time Between Memory-Set Sizes 4 and 2 (Measured in Milliseconds) at Each Combination of the Other Independent Variables-Task, Trial Type, and Display Size-in Experiment 1

\begin{tabular}{ccc}
\hline \multirow{2}{*}{$\begin{array}{c}\text { Display } \\
\text { Type }\end{array}$} & \multicolumn{2}{c}{ Trial Type } \\
\cline { 2 - 3 } & Positive & Negative \\
2 & 2-Response Task (Training) \\
4 & -6.12 & -8.13 \\
& -3.83 & -1.12 \\
2 & 3-Response Task (Transfer) & \\
4 & $46.12^{*}$ & -2.97 \\
\hline
\end{tabular}

Note-These differences were tested using the Dunn multiple comparison test $[C R(0.5,20,7)=16.13] . \quad{ }^{*} p<.01$.

Table 2

The Difference in Mean Reaction Time Between Display Sizes 4 and 2 (Measured in Milliseconds) at Each Combination of the Other Independent Variables-Task, Trial Type, and Memory-Set Size-in Experiment 1

\begin{tabular}{ccc}
\hline \multirow{2}{*}{$\begin{array}{c}\text { Memory-Set } \\
\text { Size }\end{array}$} & \multicolumn{2}{c}{ Trial Type } \\
\cline { 2 - 3 } & Positive & Negative \\
2 & 12.27 & \\
4 & 13.56 & $19.98^{*}$ \\
& 2-Response Task (Training) & $26.98 \dagger$ \\
2 & -1.28 & \\
4 & 0.86 & 12.84 \\
\hline
\end{tabular}

Note-These differences were tested using the Dunn multiple comparison test $[C R(.05,20,7)=16.13] .{ }^{*} p<.05 . \quad t p<.01$. 
Table 3

Percent Errors as a Function of Task and Trial Type in Experiment 1

\begin{tabular}{cccc}
\hline & \multicolumn{3}{c}{ Trial Type } \\
\cline { 2 - 4 } Task & Positive & Negative & Mean \\
\hline Training (2-response) & 4.41 & 3.40 & 3.91 \\
Transfer (3-response) & 7.62 & 1.52 & 4.57 \\
\hline
\end{tabular}

trial type $[F(1,7)=7.52, p<.05]$. This interaction is shown in Table 3 . There was a large increase in errors for positive trials in the 3-response task. This is not surprising due to the additional discrimination required. However, it is interesting to note that there was a concomitant decrease in error rate for negative trials in the 3-response task such that the block error rate (including both positive and negative trials) remained nearly invariant across the two tasks (2-response vs. 3-response). In general, error rates were low (in the range of $5 \%$ ). The differences in percent errors as a function of memory-set and display sizes were tested at each combination of the other independent variables using Dunn's multiple comparison technique. There were no significant differences. Thus, the RT data do not appear to reflect any speedaccuracy tradeoffs.

\section{EXPERIMENT 2}

This experiment was identical to Experiment 1 with the exception that the order of tasks was reversed. That is, the 3-response task was used for the first six training sessions and the 2-response task was used for the last three transfer sessions. This reversal-of-task order allows examination of learning in the 3-response task uncontaminated by previous experience in the 2-response task. This experimental manipulation is important in order to assess whether the performance on the 3-response task of Experiment 1 was a result of previous training (using the 2-response task) or was due simply to constraints imposed by the 3-response task.

\section{Method}

Subjects. Three male and 5 female undergraduates from the introductory psychology subject pool at The Ohio State University served as subjects. The subjects were given class credit for up to $4 \mathrm{~h}$ of participation and were then paid at the rate of $\$ 2.50 / \mathrm{h}$ for all additional hours. All subjects had normal or corrected-to-normal vision. All subjects were right-handed.

Design. The design was identical to that used in Experiment 1, with the exception that the 3-response task was used as the training task and the 2-response task was used as the transfer task.

Apparatus and Stimuli. The apparatus and stimuli were the same as in Experiment 1.

Procedure. The procedure was the same as in Experiment 1, with the exception that the task order was reversed. For the first six sessions in Experiment 2, subjects performed the 3-response/training task. For the last three sessions, the subjects performed the 2 response/transfer task.

\section{Results and Discussion}

The principal comparison involved a $2 \times 2 \times 2 \times 2$ $\times 8$ repeated measures analysis of variance. The factors were task (3-response/training vs. 2-response/transfer), trial type (positive vs. negative), display size ( 2 vs. 4 ), memory-set size ( 2 vs. 4 ), and subjects. The dependent variables were RT and percent errors. The data were collected in the manner described for Experiment 1.

Reaction time. The RT results are shown in Figure 2. Each data point represents an average across 8 subjects. As with Experiment 1, an analysis of variance yielded a significant three-way interaction between task, trial type, and memory-set size $[F(1,7)=11.18, p<.025]$. This interaction can best be understood in the context of Table 4 . In the 2-response/transfer task and for negative trials in the 3-response/training task, RT does not change as a function of memory-set size. However, there is an increase in RT as a function of memory-set size for positive trials in the 3-response/training task.

The fact that the pattern of RTs is the same for Experiments 1 and 2 suggests that the results obtained are due to constraints within the individual tasks and not a consequence of transfer between tasks. It seems that the independence of RT and memory-set size so often found in consistent mapping search results only when targets can be treated as a single equivalence class. If the task is structured so that subjects are required to make discriminations within the target set, it is likely that latency will increase with increasing memory-set size.

There was also a significant main effect for display size $[F(1,7)=108.03, p<.001]$. Mean RT was $13 \mathrm{msec}$ faster for display size 2 than for display size 4 . Although this effect was relatively small, it was consistent across subjects. All 8 subjects showed increasing average RT with increasing display size. Dunn's multiple comparison technique was used to test RT differences as a function of display size at each combination of the other variables. None of these differences was significant.

The finding that changing the task demands (2-response vs. 3-response) affected the effects of memory-set size without affecting the effects of display size suggests that

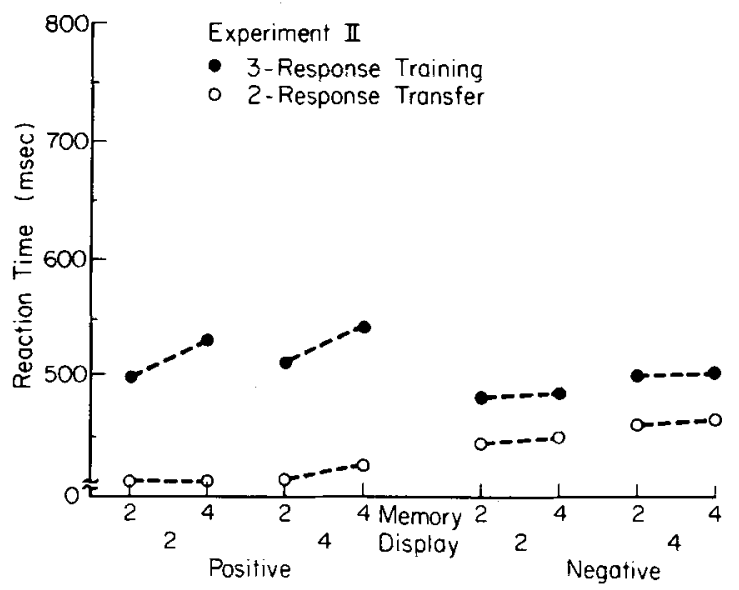

Figure 2. Reaction time data for Experiment 2. Reaction times are plotted as a function of task (3-response vs. 2-response), trial type (positive vs. negative), display size (2 vs. 4 ), and memory-set size (2 vs. 4). 
Table 4

The Difference in Mean Reaction Time (Measured in Milliseconds) Between Memory-Set Sizes 4 and 2 at Each Combination of the Other Independent Variables-Task, Trial Type, and Display Size-in Experiment 2

\begin{tabular}{ccc}
\hline \multirow{2}{*}{$\begin{array}{c}\text { Display } \\
\text { Size }\end{array}$} & \multicolumn{2}{c}{ Trial Type } \\
\cline { 2 - 3 } & Positive & Negative \\
2 & 2-Response Task (Transfer) \\
4 & 0.89 & 5.72 \\
& 12.97 & 5.14 \\
2 & $32.06^{*}$ & \\
4 & $29.61^{*}$ & 4.41 \\
\end{tabular}

Note-These differences were tested using the Dunn multiple comparison test $[C R(.05,20,7)=21.94] . \quad{ }^{*} p<.01$.

these two factors are controlled by different processes. For example, several researchers have suggested that visual search involves two processing stages (Duncan, 1980; Hoffman, 1979). Hoffman's (1979; Hoffman, Nelson, \& Houck, 1983) model is quite consistent with the results obtained in Experiments 1 and 2. Stage 1 of Hoffman's model controls display search and stage 2 controls memory search. These stages are not entirely independent in that stage 1 processing can affect the order of processing in stage 2 . Also, there is a suggestion that efficient stage 1 processing can eliminate the need for stage 2 processing in consistent mapping search tasks.

An analysis of variance comparing the changes in RT across sessions in the 3-response/training task showed an interaction between sessions, trial type, and memory-set size $[F(5,35)=2.95, p<.05]$. The effect of memoryset size on positive trials diminished with practice ( $83 \mathrm{msec}$ on Session 1 to $26 \mathrm{msec}$ on Session 6). This trend suggests that the effect of memory-set size on positive trials might disappear entirely if subjects are given enough practice. This may result from the "chunking" of the memory set into two equivalence classes reflecting the two subsets of targets.

Percent errors. An analysis of variance on percent errors resulted in a significant three-way interaction between task, trial type, and display size $[F(1,7)=8.35$, $p<.025$ ]. For positive trials in the 3-response task, there was a relatively large increase in error rate with an increase in display size from 2 to 4 . There was no such increase for either positive or negative trials in the 2response task. Nor was there an increase in error rate with display size for negative trials in the 3-response task. There were no effects of memory-set size on error rates to influence interpretation of the RT data.

\section{EXPERIMENT 3}

The results from Experiments 1 and 2 suggest that subjects adopted a two-stage decision-making strategy for performing the 3-response task. First, a decision was made as to whether a target was present or absent in the dis- play. If no target was present, then a negative response was made. The fact that response times on negative trials were independent of memory-set and display sizes indicates that the display search was parallel. If a target was present, a second decision stage was needed to determine subset membership. The fact that response times on positive trials were an increasing function of memory-set size indicates that the memory search was capacity-limited (i.e., serial or parallel dependent).

Experiment 3 was designed to test whether a similar two-stage decision-making process would be employed if the additional discrimination was contingent on distractors (nontargets) rather than targets, and if it was made on negative trials rather than on positive trials. In this experiment, subjects were first trained with a typical 2response search task and then transferred to a 3-response task. In the 3-response task, the response to a target was identical to the response used in the 2-response task. However, when no target was present, and additional discrimination was required. A particular distractor was designated as a secondary target (5 or F). If no target was present and this particular distractor was present, the subject was to respond using one key; if no target was present and this particular distractor was also not present, the subject was to respond using the other key. Thus, the threealternative responses were: (1) target present, (2) no target and $F$ or 5 present, and (3) no target and F or 5 not present.

On the basis of the previous two experiments, the following results were expected for Experiment 3. First, RT should be relatively independent of memory-set size and display size at the end of training with the 2-response task. Second, RT for positive trials in the 3-response/transfer task should also be independent of both memory-set and display sizes. Subjects should be able to use the same parallel display search used in Experiments 1 and 2 to locate a target. Since no discrimination was required within the target set, there should be no effects due to memory-set size. Finally, RT for negative trials should increase with display size. It should take longer to locate the particular distractor ( 5 or F) among other distractors of the same category (numbers or letters) as the number of distractors in the display increased. RT for negative trials should not be affected by memoryset size, since the same single secondary target was to be used throughout the experiment. Memory-set size for the secondary discrimination would be constant (1 item).

\section{Method}

Subjects. Six female and 2 male undergraduates from the introductory psychology subject pool at The Ohio State University served as subjects. The subjects were given class credit for up to $4 \mathrm{~h}$ of participation and were then paid at the rate of $\$ 2.50 / \mathrm{h}$ for all additional hours. All subjects had normal or corrected-to-normal vision. All subjects were right handed.

Design. As in the previous experiments, this experiment employed a $2 \times 2 \times 2 \times 2 \times 8$ repeated measures design. The factors were task (2-response/training vs. 3-response/transfer), trial type (posi- 
tive/target present vs. negative/no target), display size (2 vs. 4), memory-set size ( 2 vs. 4 ), and subjects. The dependent variables were RT and percent errors.

Apparatus and Stimuli. The apparatus and stimuli were the same as in Experiments 1 and 2.

Procedure. The only procedural change from Experiment 1 was that the response protocols were changed. In the 2-response task for this experiment, the subject indicated detection of a target by pressing the left key and detection of no target (a negative trial) by pressing the right key. This protocol reversal was made so that, when an additional discrimination was asked for (this time contingent on distractors), the extra response would be on the right hand, as it was in the previous experiments. The 3-response task required an identical response for the detection of a target (positive trial). However, there were two alternative responses for negative trials. If a trial was negative, the subjects were required to make an additional discrimination contingent on the presence or absence of a particular distractor ( $F$ or 5 ). The subjects trained with letters as distractors searched for $F$, and the subjects trained with numbers as distractors searched for 5 . If a trial was negative and one of the distractors was an F (or 5), the correct response was made by depressing the key under the right middle finger. If a trial was negative and none of the distractors was an F (or 5), the correct response was made by depressing the key under the right index finger.

\section{Results and Discussion}

The principal comparison involved a $2 \times 2 \times 2 \times 2$ $\times 8$ repeated measures analysis of variance. The factors were task (2-response vs. 3-response), trial type (positive vs. negative), display size ( 2 vs. 4 ), memory-set size ( 2 vs. 4 ), and subjects. The dependent variables were RT and percent errors. The data was obtained by the same method as described for Experiment 1.

Reaction time. The RT results are shown in Figure 3. Each data point represents an average across 8 subjects. Analysis of variance resulted in significant two-way interactions between task and trial type $[F(1,7)=55.43$, $p<.001]$, between task and display size $[F(1,7)=$ $13.67, p<.01]$, and between trial type and display size $[F(1,7)=13.05, p<.01]$. Thus, the difference in RT between positive and negative trials was greater for the

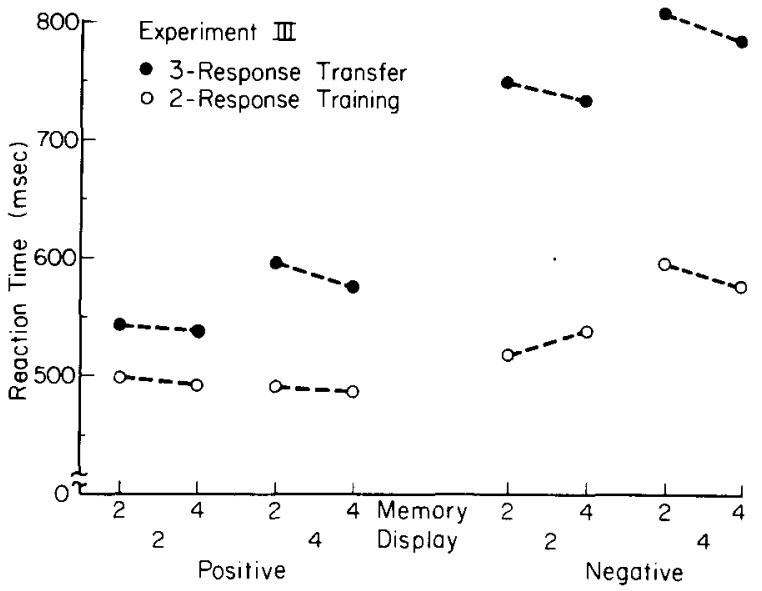

Figure 3. Reaction time data for Experiment 3. Reaction times are plotted as a function of task (3-response vs. 2-response), trial type (positive vs. negative), display size ( 2 vs. 4 ), and memory-set size ( 2 vs. 4$)$. 3-response task than for the 2-response task; there was a large effect of display size in the 3-response task and almost no effect in the 2-response task; and the effect of display size tended to be larger for negative trials than for positive trials.

This pattern of results did not conform to the expectations based on Experiments 1 and 2. The subjects did not appear to be using a sequential decision strategy in the 3-response task-first deciding whether a target was present or not and only then making the additional discriminations if required. The requirement to discriminate among the distractors seemed to have interfered with the subjects' ability to locate targets.

One hypothesis to account for this unexpected pattern of results is that there simply is not enough time for two sequential display searches-one to check for primary targets and a second to check for the secondary target. The duration of the search display ( $500 \mathrm{msec}$ ) may not be sufficient for two searches. In this case, the pattern of results may be due to constraints inherent in the structure of the 3-response task, which may require the subjects to search simultaneously for both primary and secondary targets.

An alternative hypothesis is that practice in a consistent mapping visual search task teaches subjects to ignore distractors. This ability to filter out distractors may be an important aspect of automatic detection. In a sense, the distractors fade into the background and the targets thus "pop out" of the display. Once the distractors have become part of the background (following 2-response training), it may be very difficult to make discriminations among them. Making such discriminations may require a new gestalt in which the distractors become part of the foreground, thus competing with the targets for attention and resulting in a display-size effect.

Results from transfer studies reported by Shiffrin and Dumais (1981) are consistent with this alternative explanation. They found the same positive transfer when subjects were asked to search for new targets among old distractors as when they were asked to search for old targets among new distractors. They concluded that "the learning that underlies automatic detection depends on both target accentuation (target attending) and distractor inhibition (distractor ignoring)." This phenomenon of distractor inhibition is also similar to the phenomenon of latent inhibition (Lubow, 1973). Lubow described latent inhibition as a "decrement in learning performance which results from the nonreinforced preexposure of the to-beconditioned stimulus." Conditioned attention theory ( $\mathrm{Lu}$ bow, Schnur, \& Rifkin, 1976; Lubow, Weiner, \& Schnur, 1981) attributes this inhibition to a reduction in the attention strength associated with noncontingent (distractors) stimuli.

The point appears to be that the ability to efficiently attend to certain target stimuli may, in part, depend on our ability to filter out distractions. If task demands require that we now attend to information that was previously filtered out, this new task will be more difficult and may result in competition with and perhaps disruption of 
our ability to attend to former targets. In Experiment 3, the pattern of results found in the 3-response task may reflect a learned inhibition of distractors resulting from training in the 2-response search task.

An additional analysis examined changes in RT across sessions in the 2-response training task. Average RT improved from 595 to $508 \mathrm{msec}$. However, this difference failed to reach significance $[F(5,35)=2.14, p<.10]$. It is not clear why improvement was slower in this experiment than in Experiment 1 . The only difference is that in Experiment 1 positive responses were made with the dominant right hand, whereas in this experiment positive responses were made with the nondominant left hand.

Percent errors. An analysis of variance for percent errors resulted in a significant interaction between task and trial type $[F(1,7)=14.04, p<.01]$ and between trial type and memory-set size $[F(1,7)=8.02, p<.05]$. In the 2-response task, more errors were made on positive trials, whereas in the 3-response task more errors were made on the more complex negative trials. There also was a tendency to make more errors on positive trials for memory-set size 2 than on positive trials for memory-set size 4 and to make more errors on negative trials for memory-set size 4 than on negative trials for memoryset size 2. As in the other experiments, the overall error rates were low and the pattern of errors was not in a direction that should complicate the interpretation of the RT results.

\section{EXPERIMENT 4}

In this experiment, the two tasks that were used in Experiment 3 were employed, but the order of tasks was reversed. That is, the 3-response task was used for the first six training sessions and the 2-response task was used for the last three transfer sessions. The logic for this reversal of tasks was the same as in Experiment 2. This reversal allowed performance in the 3-response task to be studied outside the context of 2-response task training. This experimental manipulation was important in order to assess whether the performance on the 3-response task of Experiment 3 was a result of previous training (perhaps resulting in inhibition of distractor processing) or due simply to constraints of the 3-response task (perhaps insufficient time to employ a two-stage search strategy).

\section{Method}

Subjects. Two male and 6 female undergraduates from the introductory psychology subject pool at The Ohio State University served as subjects. They were given class credit for up to $4 \mathrm{~h}$ of participation and were then paid at the rate of $\$ 2.50 / \mathrm{h}$ for all additional hours. All subjects had normal or corrected-to-normal vision, and all were right handed.

Design. As in the previous experiments, this experiment employed a $2 \times 2 \times 2 \times 2 \times 8$ repeated measures design. The factors were task (3-response/training vs. 2-response/transfer), trial type (positive vs. negative), display size ( 2 vs. 4 ), memory-set size ( 2 vs. 4), and subjects. The dependent variables were RT and percent errors.

Apparatus and Stimuli. The apparatus and stimuli were the same as those described for Experiment 1.
Procedure. The procedure was identical to that of Experiment 3 except that the order of tasks was reversed. The subjects performed the 3-response task for the first six training sessions and then the 2-response task for the remaining three transfer sessions.

\section{Results and Discussion}

The principal comparison involved a $2 \times 2 \times 2 \times 2$ $\times 8$ repeated measures analysis of variance. The factors were task (3-response/training vs. 2-response/transfer), trial type (positive vs. negative), display size ( 2 vs. 4 ), memory-set size ( 2 vs. 4 ), and subjects. The dependent variables were RT and percent errors. The data were collected in the same manner described for Experiment 1.

Reaction time. The RT results are shown in Figure 4. Each data point represents an average across 8 subjects. Analysis of variance resulted in a significant three-way interaction between task, trial type, and display size $[F(1,7)=18.29, p<.005]$. This interaction can best be understood in the context of Table 5 , which shows the difference in mean reaction time as a function of display size for each combination of the other variables (task, trial type, and memory-set size). Table 5 shows that the difference in mean RT as a function of display size is much greater for negative trials of the 3-response/training task than for any of the other conditions. Although there are some reliable differences in mean RT in the other conditions, these differences are not appreciably greater than the differences observed for the 2-response task in earlier experiments (e.g., $18 \mathrm{msec}$ in Experiment 1).

The pattern of results shown in Figure 4 is exactly the pattern that was predicted for Experiment 3. That is, it appears that subjects were using a sequential decision strategy, first determining whether a target was present or not and only then searching for the secondary target, if necessary. As in Experiments 1 and 2, it appears that subjects may have used a fast, parallel display search to determine if a target was present. If a target was found, then no further processing was required and the subject could respond "present." If no target was detected, then

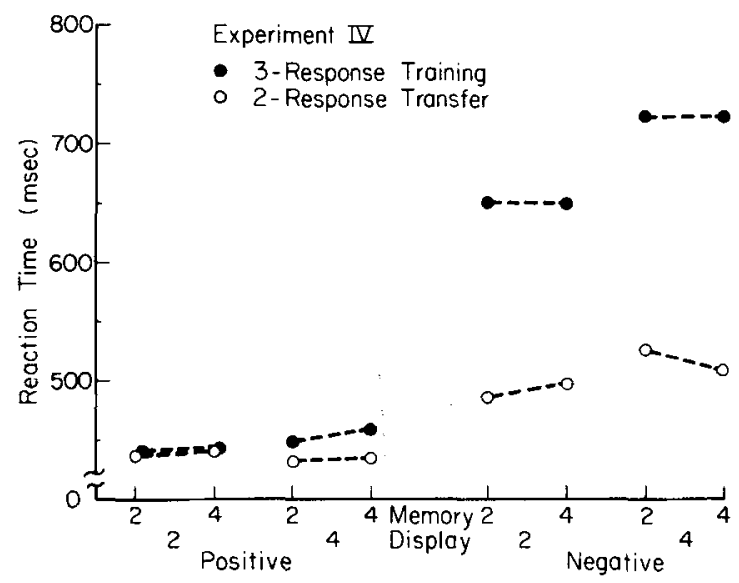

Figure 4. Reaction time data for Experiment 4. Reaction times are plotted as a function of task (3-response vs. 2-response), trial type (positive vs. negative), display size ( 2 vs. 4), and memory-set size (2 vs. 4). 
Table 5

The Difference in Mean Reaction Time (Measured in Milliseconds) Between Display Sizes 4 and 2 at Each Combination of the Other Independent Variables - Task, Trial Type, and Memory-Set Size-in Experiment 4

\begin{tabular}{ccc}
\hline \multirow{2}{*}{$\begin{array}{c}\text { Memory-Set } \\
\text { Size }\end{array}$} & \multicolumn{2}{c}{ Trial Type } \\
\cline { 2 - 3 } 2 & Positive & Negative \\
\hline & -4.52 & \\
& -4.86 & $28.94^{*}$ \\
2 & $3-$ Response Task (Transfer) & 11.45 \\
4 & 8.45 & \\
& $15.83^{*}$ & $71.75^{*}$ \\
\hline
\end{tabular}

Note-These differences were tested using the Dunn multiple comparison test $[C R(.05,20,7)=11.79] . \quad{ }^{*} p<.01$.

a slower capacity-limited search of the display was required to determine whether a secondary target was present.

The fact that the pattern of results obtained for RTs in the 3-response task differed as a function of whether or not it was preceded by 2-response task training suggests that strategies learned for the 2-response task might affect performance in the 3-response task. It is hypothesized that this strategy reflects a learned inhibition-of-distractor processing which interferes with the ability to make discriminations within these elements. The fact that only small display size effects were found in the 2-response task following 3-response task training suggests that this inhibition of distractor processing may be a normal part of "automatic processing," although it may not be required. As Shiffrin and Dumais (1981) noted, automatic processing may involve both stimulus attending and distractor ignoring. Either of these may be sufficient for RT to be relatively independent of display size.

An additional analysis examined changes in RT across sessions in the 3-response/training task. An analysis of variance resulted in an interaction between sessions, trial type, display size, and memory-set size $[F(5,35)=3.20$, $p<.025]$. This interaction is shown in Figure 5.

The improvement from Session 1 to Session 6 with the 3-response task was greater than had been seen for previous groups. This difference is primarily due to the poor performance of Experiment 4 subjects in early sessions. Early performance was particularly poor on negative trials in which the extra discrimination was required. This result suggests that the additional discrimination required on negative trials in this task was more difficult than the additional discrimination required on positive trials in the 3-response task used in Experiments 1 and 2. Despite the significant four-way interaction shown in Figure 5, the overall pattern did not seem to change much across sessions. For negative trials, RT increased with increasing display size for every session. Furthermore, the magnitude of the display size effect varied only slightly beyond the first session. For positive trials, the effects of both display size and memory-set size were small and did not vary across sessions.

Percent errors. An analysis of variance for percent errors resulted in significant two-way interactions between task and trial type $[F(1,7)=18.14, p<.005]$ and between task and memory-set size $[F(1,7)=7.30$, $p<.05]$. There were more errors on negative trials and fewer errors on positive trials for the 3-response task than for the 2-response task. However, the overall error rate for the two tasks was nearly equal. The increase in error rate for negative trials in the 3-response task is not surprising in view of the added complexity of this task. Dunn's multiple comparison procedure was used to test error differences due to memory-set and display sizes at each level of the other factors. No significant differences were found. As in the previous experiments, the pattern of errors does not seem to invalidate conclusions based on the pattern of RT data.
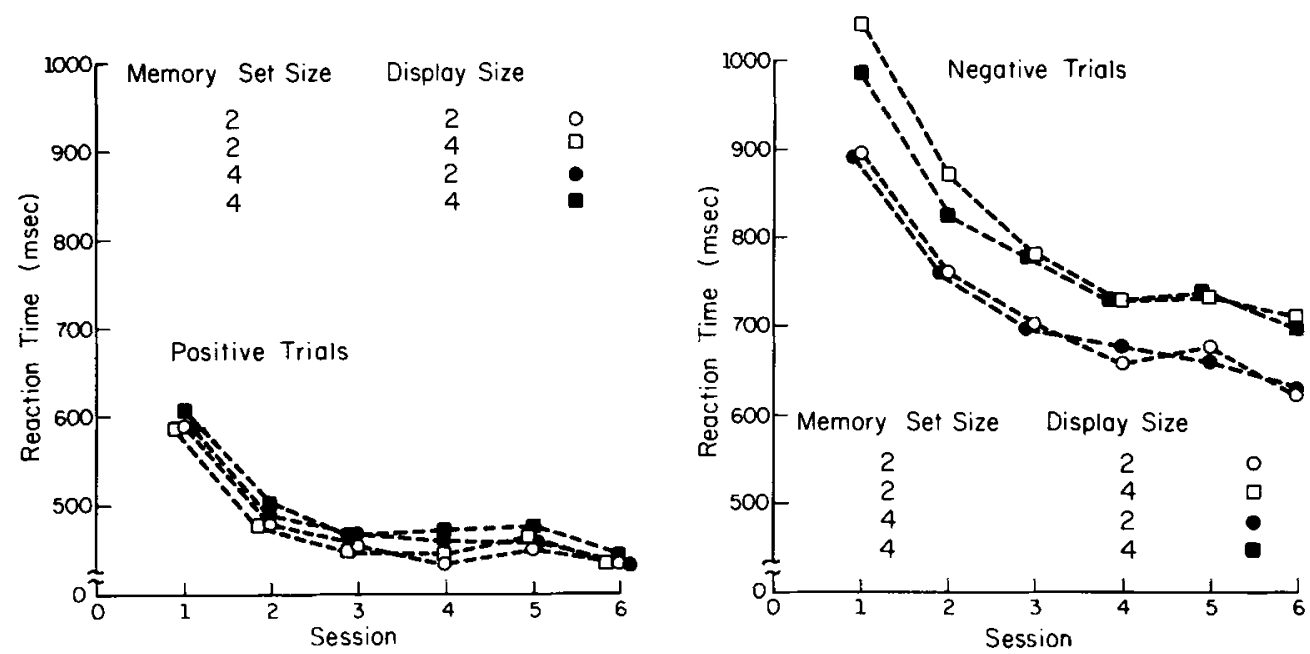

Figure 5. The interaction between sessions, trial type, display size, and memory-set size obtained in the 3response task of Experiment 4. 


\section{GENERAL CONCLUSIONS}

The primary conclusion to be drawn from this series of experiments is that, despite similarities between memory and display search, these tasks appear to involve different processing mechanisms. Although this conclusion is not new, it is an important reminder to those researchers who are manipulating both factors in a single experiment. Treating memory-set size and display size in terms of a single factor (load) may lead to inappropriate generalizations.

Memory search in these experiments is greatly affected by the ability to treat the memory set as a single equivalence class. Just as learned associations can be used to improve the capacity of working memory (Chase \& Simon, 1973; Miller, 1956), it appears that they can also be used to improve the efficiency of memory search.

Display search seems to depend, at least in part, on an ability to ignore or filter out distractors. In designing optimal filters, it is important to know both characteristics of the target signal and characteristics of the noise to be expected. This may be true for attention as well. Consistent mapping paradigms allow subjects to become thoroughly familiar with both the signal (targets) and the noise (distractors). This does not imply that distractors are identified separately, but rather that the characteristics (whether physical, acoustic, or semantic) that differentiate these items from the targets are used in tuning a cognitive filter. This filter would be similar to the "attenuator" postulated by Treisman (1969). In Experiment 3, the filter developed in the 2-response task inhibits distractor processing. Retuning this filter to search for secondary targets in the 3-response task interferes with target processing. In Experiment 4, on the other hand, the filter is developed in response to the demands of the 3-response task. Thus, it may be tuned to pass both primary and secondary targets.

The experiments in this paper do not address the important question of the relative importance of feature (physical) and categorical (semantic) distinctions in the development of the attentional filter used in display search. Even though categorically distinct targets and distractors were used in these studies, it is not clear how important this distinction is for display search. Research by Duncan (1983) and Krueger (1984) suggests that the efficiency of display search for categorically distinct stimuli may result from the average physical resemblance between targets and nontargets.

In terms of modeling performance in combination visual/memory-search tasks, the results from the experiments in this paper seem to support a two-process model such as that proposed by Hoffman (1979). In this model, display and memory search are separate, but dependent, processes. An initial display search may determine the order and perhaps even the need for processing in a second memory-search stage. Display search benefits from an ability to differentiate targets from distractors and to filter out distractors. Memory search benefits from an abil- ity to integrate targets into a single equivalence class. Practice in a consistent mapping visual search task supports both differentiation between target and distractor sets and integration within the target set.

\section{REFERENCES}

Chase, W. G., \& Simon, H. A. (1973). Perception and chess. Cognitive Psychology, 4, 55-81.

Duncan, J. (1980). The locus of interference in the perception of simultaneous stimuli. Psychological Review, 87, 272-300.

DunCan, J. (1983). Category effects in visual search: A failure to replicate the "oh-zero" phenomenon. Perception \& Psychophysics, 34, 221-232.

FitTs, P. M., \& Posner, M. I. (1967). Human performance. Monterey, CA: Brooks/Cole.

Gibson, E. J. (1969). Principles of perceptual learning. and development. New York: Appleton-Century-Crofts.

Gleitman, H., \& JoNides, J. (1976). The cost of categorization in visual search: Incomplete processing of targets and field items. Perception \& Psychophysics, 20, 281-288.

Hoffman, J. E. (1979). A two-stage model of visual search. Perception \& Psychophysics, 25, 319-327.

Hoffman, J. E., Nelson, B., \& Houck, M. E. (1983). The role of attentional resources in automatic attention. Cognitive Psychology, 51, 379-410.

Jonides, J., \& Gleitman, H. (1972). A conceptual categorization effect in visual search: $O$ as letter or digit. Perception \& Psychophysics, $12,457-460$.

Jonides, J., \& Gleitman, H. (1976). The benefit of categorization in visual search: Target location without identification. Perception \& Psychophysics, 20, 289-298.

KimBle, G. A., \& Perlmuter, L. C. (1972). The problem of volition In R. W. Christina \& L. G. Shaver (Eds.), Biological and psychological perspectives in the study of human motor performance. Dubuque: Kendal/Hunt.

KRUEGER, L. E. (1984). The category effect in visual search depends on physical rather than conceptual differences. Perception \& Psychophysics, 35, 558-564.

LABERGE, D. (1973). Attention and the measurement of perceptual learning. Memory \& Cognition, 1, 268-276.

LuBow, R. E. (1973). Latent inhibition. Psychological Bulletin, 79. 398-407.

Lubow, R. E., SchNuR, P., \& RIFKIN, B. (1976). Latent inhibition and conditioned attention theory. Journal of Experimental Psychology: Animal \& Behavioral Processes, 2, 163-174.

LUBOW, R. E., WeINER, I., \& SCHNUR, P. (1981). Conditioned attention theory. Psychology of Learning \& Motivation, 15, 1-49.

Miller, G. A. (1956). The magic number seven, plus or minus two: Some limits on our capacity for information processing. Psychological Review, 63, 81-87.

PoSNER, M. I., \& SNYDER, C. R. R. (1975). Attention and cognitive control. In R. L. Solso (Ed.), Information processing and cognition: The Loyola Symposium. Hillsdale, NJ: Erlbaum.

SCHNEIDER, W., \& Shiffrin, R. M. (1977). Controlled and automatic information processing: I. Detection, search, and attention. Psychological Review, 84, 1-66.

Shiffrin, R. M., \& Dumais, S. T. (1981). The development of automatism. In J. R. Anderson (Ed.), Cognitive skills and their acquisition. Hillsdale, NJ: Erlbaum.

SHIFFRIN, R. M., \& SCHNEIDER, W. (1977). Controlled and automatic information processing: II. Perceptual learning, automatic attending, and a general theory. Psychological Review, 84, 127-190.

Treisman, A. M. (1969). Strategies and models of selective attention. Psychological Review, 76, 282-299.

(Manuscript received January 30, 1986; revision accepted for publication April 30, 1986.) 